\title{
European perspective on the use of molecular tests in the diagnosis and therapy of thyroid neoplasms
}

\author{
Małgorzata Oczko-Wojciechowska ${ }^{1}$, Agnieszka Kotecka-Blicharz ${ }^{2}$, Jolanta Krajewska ${ }^{2}$, \\ Dagmara Rusinek ${ }^{1}$, Marcin Barczyński ${ }^{3}$, Barbara Jarząb ${ }^{2}$, Agnieszka Czarniecka ${ }^{4}$
}

${ }^{1}$ Department of Nuclear Medicine and Endocrine Oncology, Laboratory of Molecular Diagnostic and Functional Genomics, ${ }^{2}$ Department of Nuclear Medicine and Endocrine Oncology, Maria Sklodowska-Curie Institute-Oncology Center, Gliwice Branch, Gliwice, Poland; ${ }^{3}$ Department of Endocrine Surgery, Third Chair of General Surgery, Jagiellonian University Medical College, Kraków, Poland; ${ }^{4}$ The Oncologic and Reconstructive Surgery Clinic, Maria Sklodowska-Curie Institute-Oncology Center, Gliwice Branch, Gliwice, Poland

Contributions: (I) Conception and design: M Oczko-Wojciechowska, A Czarniecka; (II) Administrative support: M Oczko-Wojciechowska; (III) Provision of study materials or patients: All authors; (IV) Collection and assembly of data: All authors; (V) Data analysis and interpretation: All authors; (VI) Manuscript writing: All authors; (VII) Final approval of manuscript: All authors.

Correspondence to: Małgorzata Oczko-Wojciechowska. Department of Nuclear Medicine and Endocrine Oncology, Laboratory of Molecular Diagnostic and Functional Genomics, Maria Sklodowska-Curie Institute-Oncology Center, Gliwice Branch, Wybrzeze AK 14, 44-100 Gliwice, Poland. Email: malgorzata.oczko-wojciechowska@io.gliwice.pl.

\begin{abstract}
Thyroid nodules are frequently observed, particularly in individuals of over 60 years of age. On the other hand, most of the detected changes are benign and they do not require surgery. Therefore, differentiation between benign and malignant lesions in preoperative diagnosis is of crucial importance. Currently, the use of fine-needle aspiration biopsy (FNAB) and cytological assessment are the gold standard in the diagnosis of thyroid nodules. This procedure significantly reduces the need for diagnostic surgical intervention. However, approximately $15-30 \%$ of cytological results are classified as indeterminate. This is mainly due to the lack of specific cytomorphologic features that would facilitate the diagnosis based on cell evaluation under microscopic assessment. For the diagnoses of atypia of undetermined significance/follicular lesion of undetermined significance (AUS/FLUS), the assessment of invasion is crucial. Such an evaluation is not possible in cytology. Recently, molecular tests have been developed. They improve cytological diagnosis, particularly in the case of indeterminate results. Commercially available tests are developed based on the North American population. It is important to assess whether such tests can be used in the evaluation of e.g., European population.
\end{abstract}

Keywords: Thyroid nodules; indeterminate cytology; molecular classifier

Submitted Oct 18, 2019. Accepted for publication Oct 30, 2019.

doi: 10.21037 /gs.2019.10.26

View this article at: http://dx.doi.org/10.21037/gs.2019.10.26

\section{Introduction}

Thyroid nodules are frequently detected although their prevalence depends on the studied population and the methods used to detect them. It is estimated that $2-6 \%$ of thyroid nodules are detected by palpation whereas $19-35 \%$ are detected by ultrasound assessment. However, autopsy data indicate that the prevalence of thyroid lesions can reach even $85 \%$ (1-3). The frequency of nodule detection increases with age, particularly after 60 years of age. Of note, only about $5 \%$ of nodular lesions in the thyroid are malignant $(4,5)$. Papillary thyroid cancer (PTC) is the most frequently detected malignancy $(14.3 / 100,000$ annually in the US) (6). In the recent 4 decades there has been a steady increase in the prevalence of PTC $(5,7,8)$. Routine assessment of suspicious thyroid lesions is based on ultrasound and cytological examination using a fineneedle aspiration biopsy (FNAB). The most prestigious 
Table 1 TBSRTC and usual management after diagnosis

\begin{tabular}{lll}
\hline $\begin{array}{l}\text { Category of the } \\
\text { Bethesda system }\end{array}$ & Meaning & Usual management \\
\hline I & Non-diagnostic or inadequate & Repeat FNA with ultrasound guidance \\
II & Benign & Clinical follow-up \\
III & AUS/FLUS & Repeat FNA, molecular testing, lobectomy \\
& & (diagnostic surgery) \\
IV & Follicular neoplasm or suspicious for a follicular neoplasm & Molecular testing, lobectomy (diagnostic surgery) \\
V & Suspicious for malignancy & Lobectomy or near-total thyroidectomy \\
VI & Malignant & Lobectomy or near-total thyroidectomy \\
\hline
\end{tabular}

TBSRTC, The Bethesda System for Reporting Thyroid Cytopathology; FNA, fine-needle aspiration; AUS/FLUS, atypia of undetermined significance/follicular lesion of undetermined significance.

thyroid associations such as the American Association of Clinical Endocrinologists (AACE), the American Thyroid Association (ATA) and the European Thyroid Association (ETA) provide detailed guidelines on which nodules should be biopsied (9-11). The result of the cytological examination of the cells collected by FNAB is considered to be the most reliable in planning further clinical assessment. When malignancy is suspected, surgery is recommended, whereas in the case of benign lesions, watchful waiting strategy should be adopted.

In 2009, the National Cancer Institute in Bethesda, MD, USA proposed a classification of cells in thyroid cytology and in 2017 the criteria were revised (12). Currently, it is the basic system for reporting thyroid cytopathology that is the basis for further clinical management (Table 1). The system is known as "Bethesda" and is based on six categories, i.e., (I) nondiagnostic/inadequate, (II) benign, (III) atypia of undetermined significance/follicular lesion of undetermined significance (AUS/FLUS), (IV) follicular neoplasm or suspicious for a follicular neoplasm, (V) suspicious for malignancy, and (VI) malignant (12).

Although FNAB is a standard and well-recognized diagnostic procedure for suspicious thyroid nodules, it has certain limitations. The procedure is not easy and requires experience. Additionally, the assessment of individual cells can be difficult to interpret and poses a challenge to pathologists. It is estimated that approximately $20-30 \%$ of all thyroid nodule biopsies are classified as "indeterminate" and are then mainly assessed as Bethesda class III (13), in which the risk of thyroid cancer is $6-30 \%$ (12) and even $48 \%$ (14) and Bethesda class IV, which mainly refers to the suspicion of follicular neoplasm. Among thyroid tumors cytologically assessed as "indeterminate" that are surgically resected approximately $15-30 \%$ are malignant lesions that require surgical intervention. As a result, the majority of resected nodules are benign and do not require such a radical approach. This has a multidimensional meaning due to the fact that unnecessary surgery is related to constant hormonal treatment and an endocrine follow-up of patients throughout life. Postoperative complications may also occur, which is associated with the psychological burden of patients and a decreased quality of life. Another important issue is connected with the economic aspect of surgery and postoperative care. This means that additional diagnostic markers are needed for the above two groups of the Bethesda system classification. As a result, other independent factors could add to the cytological picture. Sensitivity to detect these lesions is also of crucial importance. Such lesions require surgical treatment due to high potential of malignancy and aggressiveness.

In the current stage of development of genetic techniques, molecular markers seem to be readily available and perfectly adopted for routine use in everyday clinical practice. Significant progress in understanding the molecular biology of thyroid cancer and particularly PTC has encouraged many scientists to develop molecular classifiers that allow them to differentiate between malignant and benign lesions. The results of the Cancer Genome Atlas Network systematized knowledge of molecular events in PTC (15) and showed that most malignant lesions are characterized by changes in the DNA. Currently, patients with cytologically indeterminate thyroid nodules (Bethesda system) usually undergo diagnostic surgery (mainly lobectomy). Therefore, the possible benefit of using a molecular classifier in cytological diagnoses of indeterminate nodules was considered in the $2^{\text {nd }}$ edition 
of The Bethesda System for Reporting Thyroid Cytopathology (TBSRTC) and in the ATA guidelines $(9,12)$. According to these recommendations, the use of molecular tests is useful to assess the risk of malignancy so that unnecessary diagnostic surgery could be avoided.

Additionally, the introduction of a new diagnosis by the WHO in 2017 is an important aspect in the diagnosis of thyroid cancer. Classification of Tumors of Endocrine Organs reclassified thyroid tumors and classified tumors of endocrine organs by introducing a new section termed "other encapsulated follicular-patterned thyroid tumors" (16). This section includes diagnoses termed uncertain malignant potentials, which include follicular tumor of uncertain malignant potential, well-differentiated tumor of uncertain malignant potential and a new diagnosis, i.e., non-invasive follicular thyroid neoplasm with papillary-like nuclear features (NIFTP) that is considered a benign lesion more similar to follicular adenoma in the clinical course of the disease (17).

NIFTP arouses great interest in relation to preoperative diagnosis. To exclude the malignancy of this tumor, it is necessary to rule out any features of invasion that cannot be assessed in biopsy material. Therefore, despite the fact that the use of FNAB of thyroid nodules has reduced unnecessary surgical treatment, it is still not a sufficient method and requires other supportive methods.

\section{Preoperative diagnosis of thyroid cancer-from a single gene to a molecular classifier}

The use of immunohistochemical (IHC) markers that assess the malignancy of lesions was the first stage of searching for markers that would increase the sensitivity of FNAB. The use of the following markers was postulated: galectin-3, HBME, fibronectin 1 or cytokeratin 19. However, they proved to be insufficient for the assessment of biopsy material (18-21). It was mainly due to the fact that the use of different antibodies and different visualization methods results in difficulties in the interpretation of the IHC. Additionally, the expression of these markers may be also detected in follicular adenomas $(22,23)$.

The increasing knowledge on molecular changes at the DNA level in thyroid cancer cells is a promising field in the search for molecular markers. Of note, knowledge related to the molecular basis of thyroid cancer was mainly related to postoperative material. Only in the last decade has there been an increase in studies using biopsy material. These studies are connected with the analysis of changes in the
DNA sequence (DNA mutations) and gene expressions (mRNA analysis).

Due to the fact that PTC is the most prevalent thyroid cancer, it is also best known in terms of molecular characteristics. The first attempts to use the selection of malignant lesions based on cytology were made using single molecular markers most commonly observed in PTC such as $B R A F$ gene mutations, RET and NTRK rearrangements (24-30) or PAX8/PPARy fusion characteristic of follicular tumors. However, these alterations do not always occur in malignant tumors and the absence of $B R A F$ mutation does not mean that the lesion has no malignant potential. $B R A F$ mutation can be an important marker determining tumor malignancy but mainly in the countries where the prevalence of this mutation is high and reaches even $83 \%$ (25). However, the prevalence of $B R A F$ mutation in European countries ranges from $50 \%$ to $70 \%(31,32)$. In addition, due to the potential tumor heterogeneity, it can occur in single cell clones and may not be detected when low-sensitivity methods are used.

For this reason, multi-gene panels were tested. As a result, the first tumor-specific mutations test was developed from seven genes most characteristic of PTC and follicular thyroid cancer, i.e., point mutations within BRAF, KRAS, HRAS and NRAS genes, and RET/PTC1, RET/PTC3 and PAX8/PPAR $\gamma$ rearrangements (33). After discovering an important role of the NTRK genes family fusion, these molecular markers were also included in the classifier (34). The analytical sensitivity of the molecular test ranged from $38 \%$ to $85.6 \%$ and the specificity ranged from $95 \%$ to $100 \%$. RAS genes mutations and PPRG/ $P A X 8$ rearrangement show the lowest sensitivity since they occur in benign and malignant lesions with a variable frequency. Of note, the first tests were performed using molecular techniques of limited sensitivity. However, it was the introduction of highly sensitive and highly effective methods such as oligonucleotide microarrays and next generation sequencing (NGS) that allowed the detection of mutations or gene expressions that were present even in small amounts.

The first gene panel for preoperative diagnostic use, defined as a molecular classifier, was presented in 2010 (35). The classifier was based on the assessment of the expression of 147 genes selected in the study using high density oligonucleotide microarrays. This study was extended by a multicenter prospective study, which involved over 3,900 patients (36).

It was the turning point for preoperative molecular 


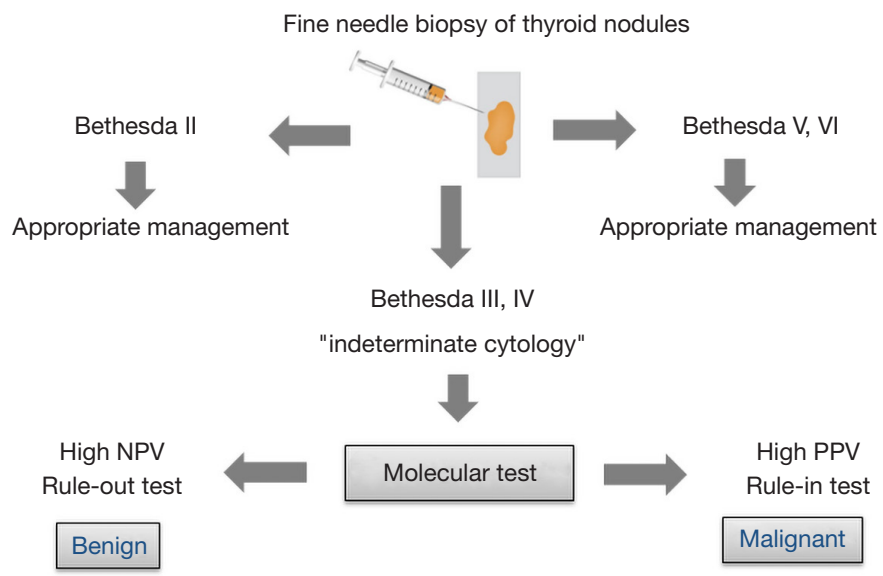

Figure 1 The use of the molecular tests in preoperative diagnosis of thyroid tumors. NPV, negative predictive value; PPV, positive predictive value.

diagnosis of thyroid tumors. The first NGS test based on the assessment of changes in the DNA sequence for 15 genes was presented in 2013 (37). NGS method allows the assessment of many gene alterations in one analysis. Next, miRNA-based classifier was developed (38). In fact, all the above classifiers form two groups of tests although all of them have the same aim, which is to distinguish between malignant and benign lesions in biopsy material. Some of these classifiers are used to exclude malignant lesions ("ruleout" test), while the others are used to confirm malignant lesions ("rule-in" test). Both types of tests are determined by two values, i.e., negative predictive value (NPV) and positive predictive value (PPV). However, the NPV value allows to assess the probability of benign lesions when the result is negative (the higher the NPV, the higher the probability of a benign lesion). PPV, on the other hand, allows to assess the probability of malignant lesions when the result is positive (the higher the PPV, the higher the probability of a malignant lesion) in a given population. The use of the "rule-in" and "rule-out" molecular tests is given in Figure 1.

\section{Commercially available molecular tests used in preoperative diagnosis of thyroid cancer-are they useful to endocrinologists and surgeons in Europe?}

Until recently, there were 4 companies on the US market that offered commercial tests for preoperative diagnosis of thyroid nodules (Veracyte Inc., CBLPath Inc., Interpace Diagnostics Inc. and Rosetta Genomics Inc.). The test developed by Rosetta Genomics Inc., after its bankruptcy, was taken over by Interpace Diagnostics Inc. (Table 2).

The first commercial molecular test for preoperative diagnosis of thyroid nodules was the Afirma gene expression classifier (GEC) test or its new version known as genomic sequencing classifier (GSC; Veracyte Inc., South San Francisco, CA, USA). This is a "rule-out" test which provides the information whether the lesion is benign or suspicious. The first version of this test (GEC) is based on the assessment of mRNA expression using high density oligonucleotide microarrays and was developed on the basis of a 2010 study by Chudova et al. (35). The test is divided into several steps.

The first step includes the assessment of the expression of 25 genes typical of metastases of other cancers to the thyroid gland (breast cancer, kidney carcinoma and melanoma) and Hürthle cell and medullary thyroid cancer (MTC). If the result is defined as "suspicious" or "MTC", the analysis is terminated and suggests metastasis to the thyroid or MTC. On the other hand, if the result is negative, the appropriate stage of the analysis is introduced, i.e., the assessment of the expression of 142 genes. At this stage, the result is either "benign" or "suspicious". When the lesion is suspicious, additional assessment of $B R A F$ gene mutation and RET/PTC1 and RET/PTC3 rearrangements is performed. Of note, the aim of the test is to confirm benign lesions (NPV of $94 \%$, sensitivity of $90 \%$; PPV is low and is of $37 \%)$. The test has also some disadvantages as malignant lesions derived from Hürthle cells cannot be assessed using this test. In 2018, the latest version of the GSC was introduced (39). It is based on the NGS technique. Both 
Table 2 Commercially available tests

\begin{tabular}{lllll}
\hline Molecular test & Methodology & Type of test & Report & Source \\
\hline Afirma GEC/GSC & Gene expression analysis (mRNA) & Rule-out & Benign/suspicious & Alexander EK, 2012 \\
ThyroSeq2/3 & Gene mutation analysis (DNA) & Rule-in/rule-out & Negative/positive & Nikiforov YE, 2015 \\
& & & Steward DL, 2018 \\
Rosetta Reveal & Gene mutation analysis (DNA) & Rule-in & Type of detected mutation & Lithwick-Yanai G, 2016 \\
ThyGenX/ThyramiR & $\begin{array}{l}\text { Gene mutation analysis (DNA) and } \\
\text { miRNA expression analysis (miRNA) }\end{array}$ & $\begin{array}{l}\text { Rule-in/rule-out when } \\
\text { both steps are performed }\end{array}$ & Negative/positive & Labourier E, 2015 \\
\hline
\end{tabular}

GEC, gene expression classifier; GSC, genomic sequencing classifier.

gene expression and mutations are assessed. The test was developed to analyze several thousand genes to better detect lesions derived from Hürthle cells. The estimated cost was $\$ 6,400$ in 2018 (40). It should be borne in mind that the Afirma test is most often validated by independent studies. Therefore, it is considered the most reliable, commercially available test (40-42). Many studies showed that the number of cases that required lobectomy was reduced by approximately $50 \%$ in the AUS/FLUS diagnoses.

ThyroSeq 2 (CBLPath Inc., Rye Brook, New York, University of Pittsburgh Medical Center, Pittsburgh, PA, USA) is the second most frequent test used in the cytological assessment. This test assesses point mutations, small insertions and deletions for 14 genes, and evaluates 42 fusion genes. The mutations used in the test are related to the biology of PTC in as much as $90 \%$ (43). The test is intended to detect malignant lesions ("rule-in") and the obtained result may be positive or negative. The positive result indicates that the lesion has a malignant potential. Interestingly, this test has high PPV (83\%) and also NPV (96\%) (sensitivity 90\%; specificity $93 \%$ ). However, it was not so widely validated by other independent studies and those which used it showed significantly lower PPV and NPV values $(44,45)$. The current version of ThyroSeq 3 is characterized by sensitivity of $94 \%$ and specificity of $82 \%$ and NPV of $97 \%$, PPV of $66 \%$ for the cancer prevalence of $28 \%$ (46) (Steward, 2018). The cost of the test is USD 4,056 (41).

The ThyGenX/ThyraMIR test (Interpace Diagnostics Inc., Parsippany, NJ, USA) has the similar characteristics. It is based on the assessment of the mutations of 5 genes, i.e., BRAF, KRAS, HRAS, NRAS, PIK3CA and 3 gene rearrangements, i.e., RET/PTC1, RET/PTC 3 and $P A X 8 /$ $P P A R G$. It is also a "rule-in" test and is characterized by high NPV (94\%) and PPV (74\%) (sensitivity 89\%; specificity $85 \%$, respectively) (38). As in the case of the ThyroSeq test, the result is either positive or negative. However, to obtain high sensitivity and specificity, it should be used in combination with second part of the test based on the assessment of miRNA expression (ThyraMIR). Of note, each step can be performed separately. However, sensitivity and specificity are then decreased. The final result of the two-step assessment shows high or low probability of malignancy. There are no independent validation tests for both assays. The cost of the test is USD 5,675 (41).

RosettaGX Reveal (Rosetta Genomics Inc., Philadelphia, Pennsylvania, USA, currently Interpace Diagnostics Group, Parsippany, NJ, USA) is another test based on miRNA expression. It is the only test which also includes the European cohort. However, it is not well known and has not undergone multicentre validation. It was found to have sensitivity of $85 \%$, specificity of $72 \%$, NPV of $91 \%$, and PPV of $59 \%$ (47). Of note, 3 pathologists were involved in the cytological assessment, including 1 referring pathologist. The estimated cost of the test was USD 3,700 in 2018 (40).

Recently, it has been announced that simple and lowcost classifiers have been developed. Those molecular tests are based on real-time polymerase chain reaction (PCR) method which supports FNAB. The tests assess mRNA or microRNA expression. However, they are still under research and cannot be commercially used $(48,49)$. Interestingly, both tests are based on South American population (Chile and Brazil).

All commercial tests are widely available in the United States. However, the most significant scientific associations are not completely ready to include them in their recommendations. The ATA clinical practice guidelines recommend a molecular test for diagnosis of indeterminate 
cytology (AUS/FLUS) after consultation with a patient who should be informed about the benefit-risk ratio (9). However, the AACE guidelines which also focus on the use of molecular tests in cytologically indeterminate thyroid nodules are limited to recommendations on performing the assessment of $B R A F$ and $R A S$ genes mutations and RET/ $P T C$ and $P A X 8 / P P R G$ rearrangements (10). However, in the case of multigene classifiers, these associations neither recommend nor prohibit them, which is related to the lack of data on a long follow-up period. On the other hand, a 2015 survey by ATA revealed that more than $50 \%$ of American clinicians used a molecular test in their daily clinical practice (50). Clinicians (endocrinologists and surgeons) choose the test themselves due to the fact that there are no clear recommendations on the use of molecular tests that support the cytological diagnosis. In the United States or high-income European countries, the costs of the tests are covered by insurance companies. However, the situation is different if the test is to be used in low-income countries where costs related to diagnostic procedures and treatment are largely covered by public health protection systems. Therefore, the most important disadvantage of commercially available tests is probably related to their price that reaches several thousand dollars. Of note, in many European countries this amount of money is equal to the cost of thyroidectomy. Additionally, there are also other limitations such as the lack of large-scale validation tests involving the European population or a too short follow-up. These limitations are stressed by European experts and therefore they are not included in European recommendations on the diagnosis of thyroid nodules (11).

No analyses of currently available molecular classifiers (commercial or scientific) have been conducted in Europe yet. Of note, there is no molecular test whose price would be affordable for all European patients or European health care systems. Admittedly, the first results of using a cost-effective (EUR 150/sample) molecular classifier in the European population was presented during the ETA Conference in 2019. However, only 109 FNABs were tested (51). The test is based on a custom Mass Array platform (PTC-MA), which allows the simultaneous detection of 13 hotspot mutations and 7 fusion genes characteristic of thyroid cancer (52).

\section{Conclusions}

Cytologically indeterminate thyroid nodules pose a great challenge to pathologists, clinicians and molecular biologists. The use of single molecular markers for thyroid cancer might help to detect malignancy in FNAB (e.g., $B R A F$ gene mutation). However, this solution is still not sufficient. The use of commercially available molecular tests significantly reduces the number of patients in whom diagnostic surgical intervention is necessary. However, validation and prospective studies are still necessary to assess the tests in other than the North American population. Additionally, the high cost of tests limits their availability in low-income countries.

\section{Acknowledgments}

We wish to thank Assistant Professor Arkadiusz Badziński DHSc for assistance in the translation of the manuscript. Funding: This work was supported by the National Centre for Research and Development project under the program "Prevention practices and treatment of civilization diseases" STRATEGMED (STRATEGMED2/267398 /4/ NCBR/2015).

\section{Footnote}

Conflicts of Interest: The authors have no conflicts of interest to declare.

Ethical Statement: The authors are accountable for all aspects of the work in ensuring that questions related to the accuracy or integrity of any part of the work are appropriately investigated and resolved.

\section{References}

1. Dean DS, Gharib H. Epidemiology of thyroid nodules. Best Pract Res Clin Endocrinol Metab 2008;22:901-11.

2. Gharib H. Changing trends in thyroid practice: understanding nodular thyroid disease. Endocr Pract 2004;10:31-9.

3. Guth S, Theune U, Aberle J, et al. Very high prevalence of thyroid nodules detected by high frequency $(13 \mathrm{MHz})$ ultrasound examination. Eur J Clin Invest 2009;39:699-706.

4. Brito JP, Yarur AJ, Prokop LJ, et al. Prevalence of thyroid cancer in multinodular goiter versus single nodule: a systematic review and meta-analysis. Thyroid 2013;23:449-55.

5. Kitahara CM, Sosa JA. The changing incidence of thyroid cancer. Nat Rev Endocrinol 2016;12:646-53.

6. Davies L, Welch HG. Current thyroid cancer trends in the United States. JAMA Otolaryngol Head Neck Surg 
2014;140:317-22.

7. Jung CK, Little MP, Lubin JH, et al. The increase in thyroid cancer incidence during the last four decades is accompanied by a high frequency of BRAF mutations and a sharp increase in RAS mutations. J Clin Endocrinol Metab 2014;99:E276-85.

8. Pellegriti G, Frasca F, Regalbuto C, et al. Worldwide increasing incidence of thyroid cancer: update on epidemiology and risk factors. J Cancer Epidemiol. 2013;2013:965212.

9. Haugen BR, Alexander EK, Bible KC, et al. 2015 American Thyroid Association management guidelines for adult patients with thyroid nodules and differentiated thyroid cancer: the American Thyroid Association Guidelines Task Force on thyroid nodules and differentiated thyroid cancer. Thyroid 2016;26:1-133.

10. Gharib H, Papini E, Paschke R, et al. American Association of Clinical Endocrinologists, Associazione Medici Endocrinologi, and European Thyroid Association Medical Guidelines for clinical practice for the diagnosis and management of thyroid nodules: executive summary of recommendations. Endocr Pract 2010;16:468-75.

11. Paschke R, Cantara S, Crescenzi A, et al. European Thyroid Association Guidelines regarding thyroid nodule molecular fine-needle aspiration cytology diagnostics. Eur Thyroid J 2017;6:115-29.

12. Cibas ES, Ali SZ. The 2017 Bethesda system for reporting thyroid cytopathology. Thyroid 2017;27:1341-6.

13. Hsiao SJ, Nikiforov Y. Molecular approaches to thyroid cancer diagnosis. Endocr Relat Cancer 2014;21:T301-13.

14. Bongiovanni M, Spitale A, Faquin WC, et al. The Bethesda system for reporting thyroid cytopathology: a meta-analysis. Acta Cytol 2012;56:333-9.

15. Cancer Genome Atlas Research Network. Integrated genomic characterization of papillary thyroid carcinoma. Cell 2014;159:676-90.

16. Lloyd RV, Osamura RY, Klöppel G, et al. WHO classification of tumours of endocrine organs. 4th ed. World Health Organization, 2017.

17. Xu B, Tallini G, Ghossein RA. Noninvasive follicular thyroid neoplasm with papillary-like nuclear features: historical context, diagnosis, and future challenges. Endocr Pathol 2017;28:128-38.

18. Renshaw AA, Haja J, Lozano RL, et al. Distinguishing carcinoid tumor from small cell carcinoma of the lung: correlating cytologic features and performance in the College of American Pathologists Non-Gynecologic Cytology Program. Arch Pathol Lab Med 2005;129:614-8.
19. Bartolazzi A, Orlandi F, Saggiorato E, et al. Galectin-3expression analysis in the surgical selection of follicular thyroid nodules with indeterminate fine-needle aspiration cytology: a prospective multicentre study. Lancet Oncol 2008;9:543-9.

20. de Matos PS, Ferreira AP, de Oliveira Facuri F, et al. Usefulness of HBME-1, cytokeratin 19 and galectin-3 immunostaining in the diagnosis of thyroid malignancy. Histopathology 2005;47:391-401.

21. Saggiorato E, De Pompa R, Volante M, et al. Characterization of thyroid "follicular neoplasms" in fineneedle aspiration cytological specimens using a panel of immunohistochemical markers: a proposal for clinical application. Endocr Relat Cancer 2005;12:305-17.

22. Faggiano A, Caillou B, Lacroix L, et al. Functional characterization of human thyroid tissue with immunohistochemistry. Thyroid 2007;17:203-11.

23. Freitas BCG, Cerutti JM. Genetic markers differentiating follicular thyroid carcinoma from benign lesions. Mol Cell Endocrinol 2010;321:77-85.

24. Nam SY, Han BK, Ko EY, et al. BRAF V600E mutation analysis of thyroid nodules needle aspirates in relation to their ultrasongraphic classification: a potential guide for selection of samples for molecular analysis. Thyroid 2010;20:273-9.

25. Kim SW, Lee JI, Kim JW, et al. BRAF V600E mutation analysis in fine-needle aspiration cytology specimens for evaluation of thyroid nodule: a large series in a BRAF V600E-prevalent population. J Clin Endocrinol Metab 2010;95:3693-700.

26. Sapio MR, Posca D, Raggioli A, et al. Detection of RET/ PTC, TRK and BRAF mutations in preoperative diagnosis of thyroid nodules with indeterminate cytological findings. Clin Endocrinol (Oxf) 2007;66:678-83.

27. Marchetti I, Lessi F, Mazzanti CM, et al. A morphomolecular diagnosis of papillary thyroid carcinoma: BRAF V600E detection as an important tool in preoperative evaluation of fine-needle aspirates. Thyroid 2009;19:837-42.

28. Xing M, Tufano RP, Tufaro AP, et al. Detection of BRAF mutation on fine needle aspiration biopsy specimens: a new diagnostic tool for papillary thyroid cancer. J Clin Endocrinol Metab 2004;89:2867-72.

29. Cheung CC, Carydis B, Ezzat S, et al. Analysis of ret /PTC gene rearrangements refines the fine needle aspiration diagnosis of thyroid cancer. J Clin Endocrinol Metab 2001;86:2187-90.

30. Chung KW, Yang SK, Lee GK, et al. Detection of 
BRAFV600E mutation on fine needle aspiration specimens of thyroid nodule refines cyto-pathology diagnosis, especially in BRAF600E mutation-prevalent area. Clin Endocrinol (Oxf) 2006;65:660-6.

31. Xing M. Molecular pathogenesis and mechanisms of thyroid cancer. Nat Rev Cancer 2013;13:184-99.

32. Rusinek D, Pfeifer A, Krajewska J, et al. Coexistence of TERT promoter mutations and the BRAF V600E alteration and its impact on histopathological features of papillary thyroid carcinoma in a selected series of polish patients. Int J Mol Sci 2018. doi: 10.3390/ijms19092647.

33. Nikiforov YE, Steward DL, Robinson-Smith TM, et al. Molecular testing for mutations in improving the fineneedle aspiration diagnosis of thyroid nodules. J Clin Endocrinol Metab 2009;94:2092-8.

34. Leeman-Neill RJ, Kelly LM, Liu P, et al. ETV6-NTRK3 is a common chromosomal rearrangement in radiationassociated thyroid cancer. Cancer 2014;120:799-807.

35. Chudova D, Wilde JI, Wang ET, et al. Molecular classification of thyroid nodules using high-dimensionality genomic data. J Clin Endocrinol Metab 2010;95:5296-304.

36. Alexander EK, Kennedy GC, Baloch ZW, et al. Preoperative diagnosis of benign thyroid nodules with indeterminate cytology. N Engl J Med 2012;367:705-15.

37. Nikiforova MN, Wald AI, Roy S, et al. Targeted nextgeneration sequencing panel (ThyroSeq) for detection of mutations in thyroid cancer. J Clin Endocrinol Metab 2013;98:E1852-60.

38. Labourier E, Shifrin A, Busseniers AE, et al. Molecular testing for miRNA, mRNA, and DNA on fine-needle aspiration improves the preoperative diagnosis of thyroid nodules with indeterminate cytology. J Clin Endocrinol Metab 2015;100:2743-50.

39. Patel KN, Angell TE, Babiarz J, et al. Performance of a genomic sequencing classifier for the preoperative diagnosis of cytologically indeterminate thyroid nodules. JAMA Surg 2018;153:817-24.

40. Nishino M. Molecular cytopathology for thyroid nodules: a review of methodology and test performance. Cancer Cytopathol 2016;124:14-27.

41. Nishino M, Nikiforova M. Update on molecular testing for cytologically indeterminate thyroid nodules. Arch Pathol Lab Med 2018;142:446-57.

42. Vargas-Salas S, Martínez JR, Urra S, et al. Genetic testing for indeterminate thyroid cytology: review and metaanalysis. Endocr Relat Cancer 2018;25:R163-77.

43. Nikiforov YE, Carty SE, Chiosea SI, et al. Highly accurate diagnosis of cancer in thyroid nodules with follicular neoplasm/suspicious for a follicular neoplasm cytology by ThyroSeq v2 next-generation sequencing assay. Cancer 2014;120:3627-34.

44. Valderrabano P, Khazai L, Leon ME, et al. Evaluation of ThyroSeq v2 performance in thyroid nodules with indeterminate cytology. Endocr Relat Cancer 2017;24:127-36.

45. Toraldo G, Godley FA, Cerda SR, et al. Large independent prospective study to evaluate the performances of ThyroSeq2 multigene next generation sequencing panel analysis on cancer diagnosis in thyroid nodules with indeterminate cytopathology. Thyroid 2016;26:A148.

46. Steward DL, Carty SE, Sippel RS, et al. Performance of a multigene genomic classifier in thyroid nodules with indeterminate cytology. JAMA Oncol 2019;5:204-12.

47. Lithwick-Yanai G, Dromi N, Shtabsky A, et al. Multicentre validation of a microRNA-based assay for diagnosing indeterminate thyroid nodules utilising fine needle aspirate smears. J Clin Pathol 2017;70:500-7.

48. González HE, Martínez JR, Vargas-Salas S, et al. A 10-gene classifier for indeterminate thyroid nodules: development and multicenter accuracy study. Thyroid 2017;27:1058-67.

49. Santos MT Dos, Buzolin AL, Gama RR, et al. Molecular classification of thyroid nodules with indeterminate cytology: development and validation of a highly sensitive and specific new miRNA-based classifier test using fineneedle aspiration smear slides. Thyroid 2018;28:1618-26.

50. Burch HB, Burman KD, Cooper DS, et al. A 2015 survey of clinical practice patterns in the management of thyroid nodules. J Clin Endocrinol Metab 2016;101:2853-62.

51. Pogliaghi G, Colombo C, Muzza M, et al. Molecular testing by the custom PTC MASS array genotyping platform in thyroid fine needle aspirate. Eur Thyroid J 2019;8:8.

52. Pesenti C, Muzza M, Colombo C, et al. MassARRAYbased simultaneous detection of hotspot somatic mutations and recurrent fusion genes in papillary thyroid carcinoma: the PTC-MA assay. Endocrine 2018;61:36-41.

Cite this article as: Oczko-Wojciechowska M, KoteckaBlicharz A, Krajewska J, Rusinek D, Barczyński M, Jarząb B, Czarniecka A. European perspective on the use of molecular tests in the diagnosis and therapy of thyroid neoplasms. Gland Surg 2020;9(Suppl 2):S69-S76. doi: 10.21037/gs.2019.10.26 